\title{
New road anomaly detection and characterization algorithm for autonomous vehicles
}

\author{
H. Bello-Salau \\ Department of Telecommunication Engineering, \\ Federal University of Technology, Minna, Nigeria \\ A.M. Aibinu \\ Department of Mechatronics Engineering, \\ Federal University of Technology, Minna, Nigeria \\ A.J. Onumanyi and E.N. Onwuka \\ Department of Telecommunication Engineering, \\ Federal University of Technology, Minna, Nigeria \\ J.J. Dukiya \\ Department of Transport Management, \\ Federal University of Technology, Minna, Nigeria, and \\ H. Ohize \\ Department of Electrical/Electronics Engineering, \\ Federal University of Technology, Minna, Nigeria
}

\begin{abstract}
This paper presents a new algorithm for detecting and characterizing potholes and bumps directly from noisy signals acquired using an Accelerometer. A wavelet transformation based filter was used to decompose the signals into multiple scales. These coefficients were correlated across adjacent scales and filtered using a spatial filter. Road anomalies were then detected based on a fixed threshold system, while characterization was achieved using unique features extracted from the filtered wavelet coefficients. Our analyses show that the proposed algorithm detects and characterizes road anomalies with high levels of accuracy, precision and low false alarm rates.
\end{abstract}

Keywords Accelerometer, Bumps, Potholes, Road anomaly, Scale space filter, Wavelet transform

Paper type Original Article

(C) H. Bello-Salau, A.M. Aibinu, A.J. Onumanyi, E.N. Onwuka, J.J. Dukiya and H. Ohize. Published in Applied Computing and Informatics. Published by Emerald Publishing Limited. This article is published under the Creative Commons Attribution (CC BY 4.0) license. Anyone may reproduce, distribute, translate and create derivative works of this article (for both commercial and non-commercial purposes), subject to full attribution to the original publication and authors. The full terms of this license may be seen at http://creativecommons.org/licences/by/4.0/legalcode

Publishers note: The publisher wishes to inform readers that the article "New road anomaly detection and characterization algorithm for autonomous vehicles" was originally published by the previous publisher of Applied Computing and Informatics and the pagination of this article has been subsequently changed. There has been no change to the content of the article. This change was necessary for the journal to transition from the previous publisher to the new one. The publisher sincerely apologises for any inconvenience caused. To access and cite this article, please use Bello-Salau, H., Aibinu, A.M., Onumanyi, A.J., Onwuka. E.N. Dukiya, J.J., Ohize, H. (2020), "New road anomaly detection and characterization algorithm for autonomous vehicles", Applied Computing and Informatics. Vol. 16 No. 1/2, pp. 223-239. The original publication date for this paper was 19/05/2018.
New road anomaly detection for vehicles

Received 9 February 2018 Revised 15 April 2018 Accepted 5 May 2018 
$\mathrm{ACI}$

$16,1 / 2$

\section{Introduction}

Autonomous vehicles are vehicles capable of sensing their environment and navigating without human input under different terrains, particularly over asphalt roads. The capacity to autonavigate greatly depends on the vehicle's ability to monitor and accurately interpret road surface conditions [1]. Thus, developing effective road surface monitoring mechanisms will greatly improve the viability of autonomous vehicles, while contributing to the reduction of related road accidents around the world [2-7].

In monitoring road surfaces, accelerometers are frequently proposed for use in autonomous vehicles [8,2,9-11]. An accelerometer is a device that measures the acceleration of a body (for example, a vehicle, an aircraft or a rocket) relative to g-force [12]. The output of an accelerometer can be viewed as a series of time sampled measurements influenced by the movement of an observed body (a vehicle in this case) in three dimensional space. However, a familiar and widespread problem with processing measurements from an accelerometer lies in the noisy nature of the measurements [2]. The randomness in acceleration signals often makes road anomaly detection a difficult process, while rendering characterization an even more difficult and sometimes impossible task [3-6]. In this case, we refer to detection as the identification of an anomaly, while characterization is defined as the recognition of the type of sensed anomaly (in this case if it is either a pothole or a bump). Thus, providing new methods for improving road anomaly detection and characterization motivated the work reported in this paper.

In this regard, we propose a Road Anomaly Characterization Algorithm (RACA) based on the Wavelet Transformation Scale Space Filtering (SSF) algorithm. The SSF algorithm processes the acceleration signals by decomposing them into multiple scales. It proceeds by correlating the decomposed wavelet coefficients of the signal across adjacent scales and then filtering the noisy samples via a well-designed spatial filter. The SSF algorithm feeds a Road Anomaly Detection Algorithm (RADA) to detect road anomalies via a fixed threshold technique, while feeding RACA to characterize road anomalies. RACA achieves characterization using two unique features to differentiate potholes from bumps. Following our new approach, it is noted that the following contributions have been made:

(1) A new approach for road anomaly detection and characterization has been developed based on the application of Wavelet Transformation theory. To the best of the author's knowledge, this investigation involving the use of the WT based filter for characterizing accelerometer based dataset marks the first attempt in this regard. The results obtained also indicate positive strides in the effort towards addressing the characterization problem for future deployment in autonomous vehicles.

(2) Based on the adopted WT approach, new features were arrived at that can be used to characterize the presence of bumps and potholes on asphalt roads. One important note is that these features were only made extractable following the use of the approach proposed in our work.

The rest of this paper is structured as follows: related works are presented in Section 2, Section 3 presents the proposed methodology, while results, analysis and discussions are presented in Section 4, while conclusion is drawn in Section 5.

\section{Related works}

We briefly discuss related works on road anomaly detection while highlighting the limited consideration for solving the road anomaly characterization problem in the literature, particularly within the scope of the author's knowledge. In [13], an accelerometer was used to acquire acceleration signals across different roads. A Support Vector Machine (SVM) learning technique was used to analyze the acceleration signals to detect road anomalies and 
to determine their location. Similarly, the authors in [14] used a machine learning approach to classify road surfaces into either smooth or rough roads using signals obtained from a mobile phone based accelerometer and a gyroscope framework. While they were able to detect road anomalies with a relatively high precision rate, the anomaly characterization problem was not considered. Furthermore, the learning approaches in $[14,13]$ both require a training process leading to an increase in the complexity of these approaches, and great dependence on the specific type of input data being considered.

In [15], data was acquired using a gyroscope, an inertial sensor and a mobile phone to detect road anomalies. Authors analyzed signals using the wavelet transformation technique alongside the SVM. Specific statistics such as the absolute mean, standard deviation, variance and the energy of the signal were used to train the SVM to classify road anomalies into either severe, mild or span levels. Gonzalez et al. [16], in a similar approach used the Multilayer Perceptron (MLP) algorithm to analyze road anomalies. They demonstrated a high level of precision and detection accuracy in their experiments.

An accelerometer and a GPS based pattern recognition system was used for road anomaly detection in [17]. The process included a pre-processing stage, classification using an SVM, and visualization of the output. Results indicated better statistical performance than the other approaches considered in [16]. In [18], a GPS, video module and acceleration signals were used by an onsite real time algorithm for pothole detection. The goal was to minimize the amount of data transmitted to a central server.

A pothole patrol system comprising of a vehicle equipped with an accelerometer sensor for monitoring and detecting potholes was developed for anomaly detection in [19]. The accelerometer data captured during the drive tests were relayed via $\mathrm{WiFi}$ to a central database where it was used to train an SVM for clustering the measured acceleration data. Similarly, an approach was proposed for processing measurements obtained from a laser profilometer in [20] towards profiling road surfaces. Results indicated that the Discrete Wavelet Transformation (DWT) technique performed well in profiling the road surface than the Power Spectral Density (PSD) approach.

A crowdsourcing-based pavement monitoring system was proposed in [21] for road anomaly detection using an accelerometer, a GPS and a smartphone probe car application. A grid-based clustering algorithm was further introduced for comprehensive information extraction and filtering. Similarly, an investigation was conducted in a vehicle probe-based pavement maintenance project to examine the possibility of using data from connected vehicles for road pavement conditions monitoring [22]. Experimental results showed that pothole anomalies were detected based on a developed sigma threshold and a wavelet tranformation algorithm. Nevertheless, it was noted that the characterization problem was not considered.

Essentially, other works identified in [23,24,10,25,2] considered issues pertaining to detection, monitoring, localization, and evaluation of road anomalies, with little or no consideration for road anomaly characterization. We suggest that the difficulty associated with extracting distinct features from apparently indistinguishable (often noisy) acceleration signals may well justify this limited consideration. Thus, we consider the characterization problem in this paper. We note that effective road anomaly characterization will improve piloting in both manned and unmanned vehicle navigation. Furthermore, realtime knowledge of road conditions will facilitate quick maintenance response by particular agencies.

\section{Development of the proposed system}

Figure 1 represents the overall design for our proposed system. A discussion of each block is provided in the following sub-sections. 


\section{ACI \\ $16,1 / 2$}

\section{6}

Figure 1.

Block diagram of the road anomaly detection, characterization and profiling system.
Figure 2.

Components of the data acquisition stage.

\subsection{Input stage: road surface sensing}

In this section, we describe the development of our data acquisition system. The process of road surface sensing is shown in Figure 2a. The three major tasks in this phase include the road surface data acquisition process using an Accelerometer, the input parameter initialization, and data display for visual inspection/analysis.

We note that NI MyRio (1950) accelerometer was programmed using LABVIEW for the data acquisition exercise. A sampling rate of $30 \mathrm{~Hz}$ was set in the LABVIEW before commencing acquisition via several drive tests. The accelerometer was mounted and fastened to the dashboard of a Toyota Siena Space Bus 2009 model, a Peugeot 4062000 model and a Toyota Pontiac 2003 model all driven at a speed of $25 \mathrm{~km} / \mathrm{h}$. The choice of the dashboard for placement of the device was motivated by similar adaptations identified in $[21,19,11]$ in which the dashboard was reported as convenient position to place the accelerometer. It was ensured that the accelerometer was firmly fitted to prevent any form of loose contacts and stray vibrations. Other specific details about the experimental setup used for the data acquisition and processing can be found in [2].

We incorporated a Global Positioning System (GPS) into our design to locate road anomalies. To do this, we programmed an Arduino microcontroller under the Arduino Integrated Development Environment (IDE). The GPS data acquisition process is shown in Figure 2(b). Essentially, the system initializes the GPS module to log the time of data capture, and then records the corresponding longitude and latitude coordinates of the location where data was captured. The GPS sampling rate was set at $1 \mathrm{~Hz}$ which was found sufficient enough to provide location data points during the drive test $[21,19]$.

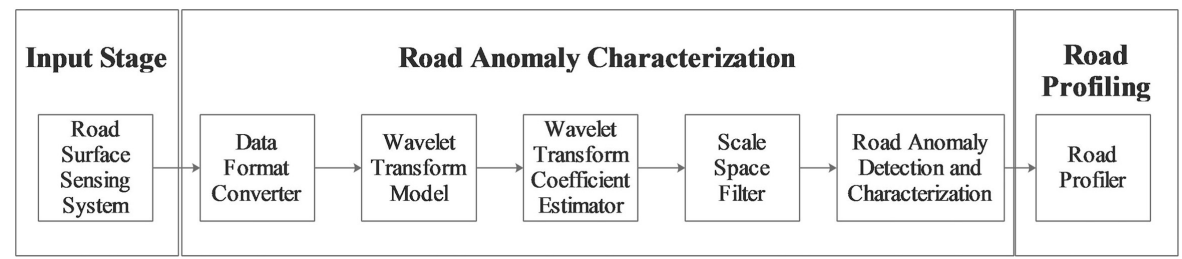

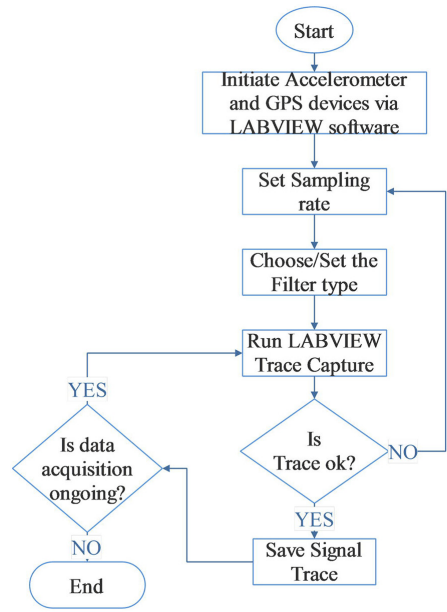

(a) Road Surface Sensing Process

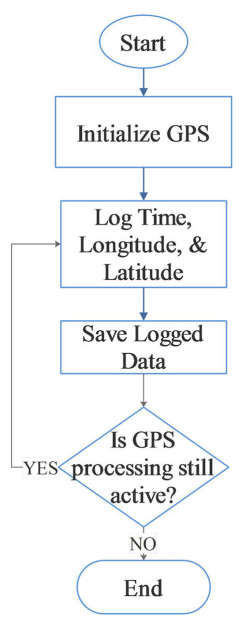

(b) GPS Data Logging Process

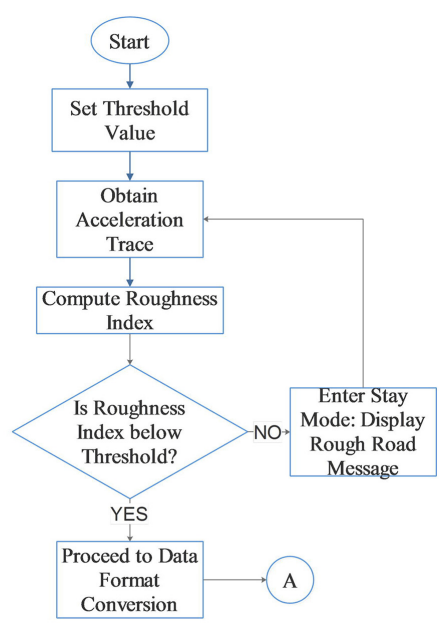

(c) Road Roughness Computing Process 
We used the standard deviation of the acceleration signal acquired over a specific period as an approximate roughness index to describe road quality. Based on this roughness index, we developed a Road Roughness Assessment Algorithm (RRAA) presented in Figure 2c to determine the smoothness of the road surface. If the roughness index value is below a certain fixed threshold value, RRAA decides that the road surface is smooth (implying that the vehicle is on an asphalt road), and thus activates the anomaly detection and characterization algorithm (described in the next subsections). However, if the index value is above the threshold, the algorithm enters a stay mode (noting that the road being plied may not be an asphalt road and thus typically defective). The program displays a "Rough Road" message during this stay period. The road anomaly detection and characterization algorithm is only activated when RRAA measures a roughness index below the threshold value.

\subsection{Processing stage: road anomaly characterization}

Our characterization algorithm employs the Wavelet Transformation (WT) theory and a noise filtering technique to characterize road anomalies into either potholes or bumps. We used the filtering technique in $[26,27]$ to filter noise from acceleration signals noting that noisy samples have lower correlation values across an increasing WT scale. This filtering technique was adopted because it processes signals along both frequency and time scales, which enables us to better resolve signals with hidden features. These hidden features can then be used for both detection and characterization purposes. We present details of each subsystem comprising the processing stage as follows:

3.2.1 Data format converter. LABVIEW software was used to program our Accelerometer. We considered the $z$-axis measurement of the Accelerometer as it directly relates to the vertical displacement of the vehicle producing different responses as the vehicle either descends into a pothole, or ascends over a bump. The signal from the Accelerometer obtained from point " $\mathrm{A}$ " in Figure 2(c) is normally outputted by the LABVIEW software in ".xml" format, which we converted to a suitable MATLAB format (".mat") for further processing. MATLAB software was used for the development of our algorithms. Basically, data is transposed from a column to a row vector and then raised to an appropriate binary power of two, and zero padded if the data size is less than the exponent value. The output of this conversion process is then passed to the characterization algorithm described in subsequent subsections.

3.2.2 Wavelet transformation model. Wavelet Transformation (WT) theory was adopted in our design. Interestingly, it is noted that while some other filtering theories may have been used only for anomaly detection purposes, the use of the WT based filter for characterization has remained to be investigated. Thus, in our work, it was hypothesized that by adopting the WT theory for filtering of acceleration signals, it will be possible to examine acceleration signals in both its frequency and timing dimensions. Our hypothesis followed the notion that by considering the timing domain of the signal in addition to its frequency dimension, we will be provided with a new scale for revealing unknown and hidden features of the signal. The discrete WT model used is expressed in Eq. (1). First, we visually examined different function patterns to determine a suitable and appropriate basis function, $\Psi_{j, k}(x)$ to be used. The particular functions that we considered are the Mexican Hat (Morlet), Haar, Daubechies (db), Coiflet and the Meyer wavelet functions. The patterns of these functions were visually compared to typical acceleration signals with an intention to choose the most similar. We found the $\mathrm{db} 2$ wavelet basis function to be the best choice. Nevertheless, these functions were analysed and their performances are reported in the result section. The discrete WT model we adopted is typically expressed as

$$
f(x)=\sum_{j=1}^{J} \sum_{k=1}^{K} \Psi_{j, k}(x) W(j, k)
$$


$\mathrm{ACI}$

$16,1 / 2$

\section{8}

where $\Psi_{j, k}(x)$ is the normalized dual basis function, $J$ is the maximum number of decomposition scales, $K$ is the maximum position in the original signal scale, $W(j, k)$ is the actual scale space decomposition at different resolution scales, $j$ is the index of the scales, and $k$ is the index position in the original signal space. Several members of the family of Daubechy functions were implemented according to Eq. (2) given as

$$
\Psi_{a, t}(x)=\frac{1}{\sqrt{a}} \Psi\left(\frac{x-t}{a}\right) \quad \text { for } a \neq 0, t \in R
$$

where $a$ is the dyadic scale parameter, $\frac{1}{\sqrt{a}}$ is the resolution, and $t=k a$ is the dyadic translation parameter. The scale variables were discretized, dilated and translated to obtain the dyadic WT using

$$
\Psi_{j, k}(x)=\frac{1}{\sqrt{2^{j}}} \Psi\left(\frac{x-k}{2^{j}}\right)
$$

where $k$ is the translation index $(0, \pm 1, \pm 2, \ldots)$. We describe how these functions in (1)-(3) were used in our approach for road anomaly characterization.

3.2.3 Wavelet transform coefficient estimator. WT coefficients, $f(x)$, are typically expressed as

$$
W(j, k)=\int_{-\infty}^{\infty} f(x) \Psi_{j, k}(x) d x
$$

where $\Psi_{j, k}(x)$ corresponds to the function values computed in (3). By increasing the scale factor, $j$, the coefficient values either remain constant or increase as the signal edges become positively correlated. On the other hand, noise samples tend to reduce at higher scales because they are poorly correlated. Using these properties, we easily extracted the noise components from the portions of interests corresponding to the anomalous points in the dataset. We describe the filtering process next based on the estimated coefficient values.

3.2.4 Scale space filter. We implemented the scale space filtering (SSF) algorithm according to $[27,28]$ for noise filtering. The SSF algorithm correlates the wavelet coefficients by directly multiplying them across adjacent scales. This can be modeled as

$$
\Omega_{j}(j, k)=\prod_{i=0}^{J-1} W(j+i, k)
$$

where $\Omega$ denotes the spatial correlation function, $J$ is the number of multiplication scales, and $W(j+i, k)$ is the $(j+i)$ th wavelet coefficients at the $k^{\text {th }}$ scale. Following (5), noise samples are identified and isolated at higher scales based on their low correlation values. This process is explained considering the first and second scale wavelet transform coefficients to be $W(1, k)$ and $W(2, k)$, respectively. Then, these two scales are multiplied for $j=1$ in (5) as follows

$$
\Omega_{2}(1, k)=W(1, k) \cdot W(2, k)
$$

To improve detection, we found it appropriate to use $\Omega_{2}(1, k)$ rather than either $W(1, k)$ or $W(2, k)$ because it produces larger responses across increasing scales. The SSF algorithm based on $\Omega_{2}(1, k)$ is described as follows:

1. Compute $W(j, k)$ and $\Omega_{2}(j, k)$ using (4) and (5) respectively.

2. each scale, the power of the correlated signal $\Omega_{2}(j, k)$ is rescaled to the wavelet coefficient at that scale $W(j, k)$.

3. An edge is identified if the absolute value of $\Omega_{2}(j, k)$ is greater than or equal to the absolute value of $W(j, k)$ when compared at the scale $(j, k)$. 
4. The edge position and its corresponding $W(j, k)$ values are stored.

5. All identified edges from $\Omega_{2}(j, k)$ and $W(j, k)$ are extracted. After extracting the first round of edges at position $(j, k)$, the samples left in $\Omega_{2}(j, k)$ and $W(j, k)$ are denoted as $\Omega_{2}^{\prime}(j, k)$ and $W^{\prime}(j, k)$.

6. The nextedge in the acceleration signal is extracted from $W(j, k)$ and $\Omega_{2}(j, k)$ by rescaling the power of $\Omega_{2}^{\prime}(j, k)$ to that of $W^{\prime}(j, k)$, and then comparing their absolute values.

7. Steps 2 to 6 is iterated until the power of the unextracted samples in $W(j, k)$ at the scale points $(j, k)$ are approximately equal.

8. The final filtered signal $W_{\text {new }}(j, k)$ is obtained after step 7 as input to the road anomaly detector and characterization algorithm, which we describe next.

3.2.5 Road anomaly detection algorithm. Our Road Anomaly Detection Algorithm (RADA) monitors the signal edges in the denoised signal to detect points corresponding to road anomalies in the signal. Transient fluctuations in the filtered signal are removed and saved as $N S D$. The absolute value of $N S D$ is computed as $N S$. A counter is created to keep track of the number of detected road anomalies in each signal window, winsig, which updates as the algorithm moves across the length of the signal. A threshold value, $T h$, is set to determine when an anomaly is detected. In each winsig, an anomaly is declared if the value of $W_{\text {new }}(j, k)$ is greater than $T h$. Thus, if one or more points in $N S$ are greater than or equal to $T h$, the algorithm normalizes the number of detected anomalies within the windowed set to 1 (and increments the anomaly count). It keeps comparing each sample within winsig to Th until the whole data length is processed. If no anomaly is detected, RADA outputs a 'NO ANOMALY' message, and reinitializes a new window to continue the detection process. The process of anomalies detection by the proposed RADA is summarized in Algorithm 1.

Algorithm 1. Road anomaly detector.

Inputs: sigden, the denoised signal from the wavelet scale space filter; $T h$, the threshold value; winsig, window size for detection;

Outputs : numofanomaly, the number of detected anomalies

1: Determine the length of the final filtered output signal (sigden) from the SSF algorithm

2: Remove transient fluctuations in the filtered signal and save as $N S D$

3: Compute the absolute value of set NSD and save as NS

4: Determine the length of $N S$ and save in $L P$

5: Set a threshold value $T h$ for anomaly detection

6: Create a counter to count the number of detected road anomaly

7: Set the window size winsig for road anomaly detection

8: Apply the set threshold value $T h$ for anomaly detection

9: An anomaly is declared if in each winsig the value of $W_{\text {new }}(j, k)$ is greater than $T h$, update the counter as the algorithm operates across the length of the signal

10: If one or more points in NS are greater than or equal to $T h$, normalizes the number of detected anomalies within the windowed set to 1 (and increment the anomaly count).

11: Compare each sample within winsig to $T h$ until the whole data length is processed

12: If no anomaly is detected, RADA outputs a 'NO ANOMALY' message, and reinitializes a new window to continue the detection process. 
$\mathrm{ACI}$

$16,1 / 2$
3.2.6 Road anomaly characterization algorithm. Our Road Anomaly Characterization Algorithm (RACA) is summarized in Algorithm 2. RACA begins by setting the size of the window, winsig, for processing $N S$ (supplied by RADA). It uses winsig to create a variable, $N S D$, which contains zeroes up to length $N S$. A sample index counter with its value initialized to 1 was created. At the start, RACA initializes the number of characterized anomalies to 0 (see lines 5 of Algorithm 2). It checks each sample in winsig (in Line 6) and then sample values less than 0 with an immediate sample value greater than or equal to 0 are characterized as bumps. Likewise, if the immediate sample value is less than 0, then RACA characterizes the sample as pothole. The counter is incremented after processing the entire samples in NSD and then RACA reinitiates to continue the characterization process.

Algorithm 2. Road anomaly characterization.

\subsection{Road Surface Profiler}

The road surface profiler system was developed to profile road surfaces using the GPS coordinates obtained from the data acquistion stage (in Section III-A). The Profiler provides a map of the different anomalous points existing along the road surface. It comprises of a Hyper Text Mark-up Language script (HTML) written and interfaced with Google map. The outputs from the HTML and Google map scripts are visualized using a web browser. The inputs for the longitude and latitude coordinates of the vehicle's location are made available via the browser's interface. The Profiler provides drivers with a view of the road surface condition to enhance their driving process.

\section{Results and discussion}

The performance of the SSF, RADA and RACA algorithms are examined and discussed in this section. Ultimately, the goal of the results presented in this section is to demonstrate the capability of our algorithms to accurately detect and characterize road anomalies (with emphasis on characterization). Furthermore, in the tabular presentation of our results, the false alarm rate, accuracy, and the precision rate are reported for the anomaly detection capability of the algorithm, while the characterization results are reported in terms of the identification of either bumps or potholes. 


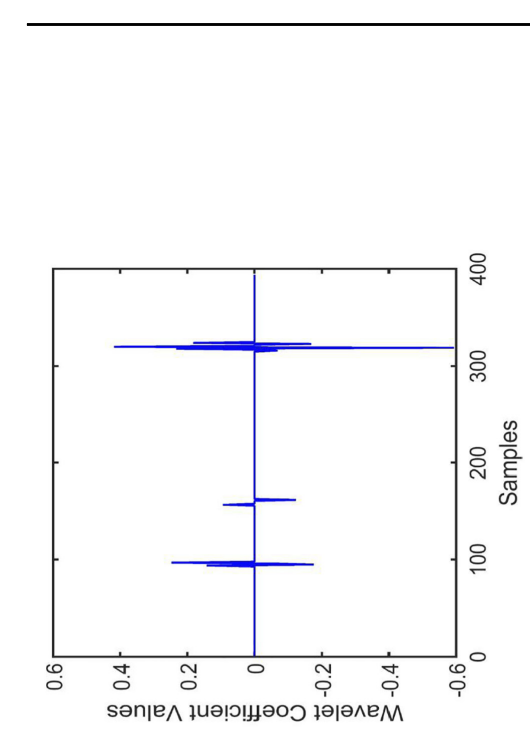

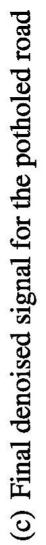

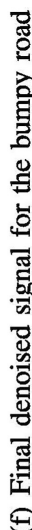

New road anomaly detection for vehicles

231
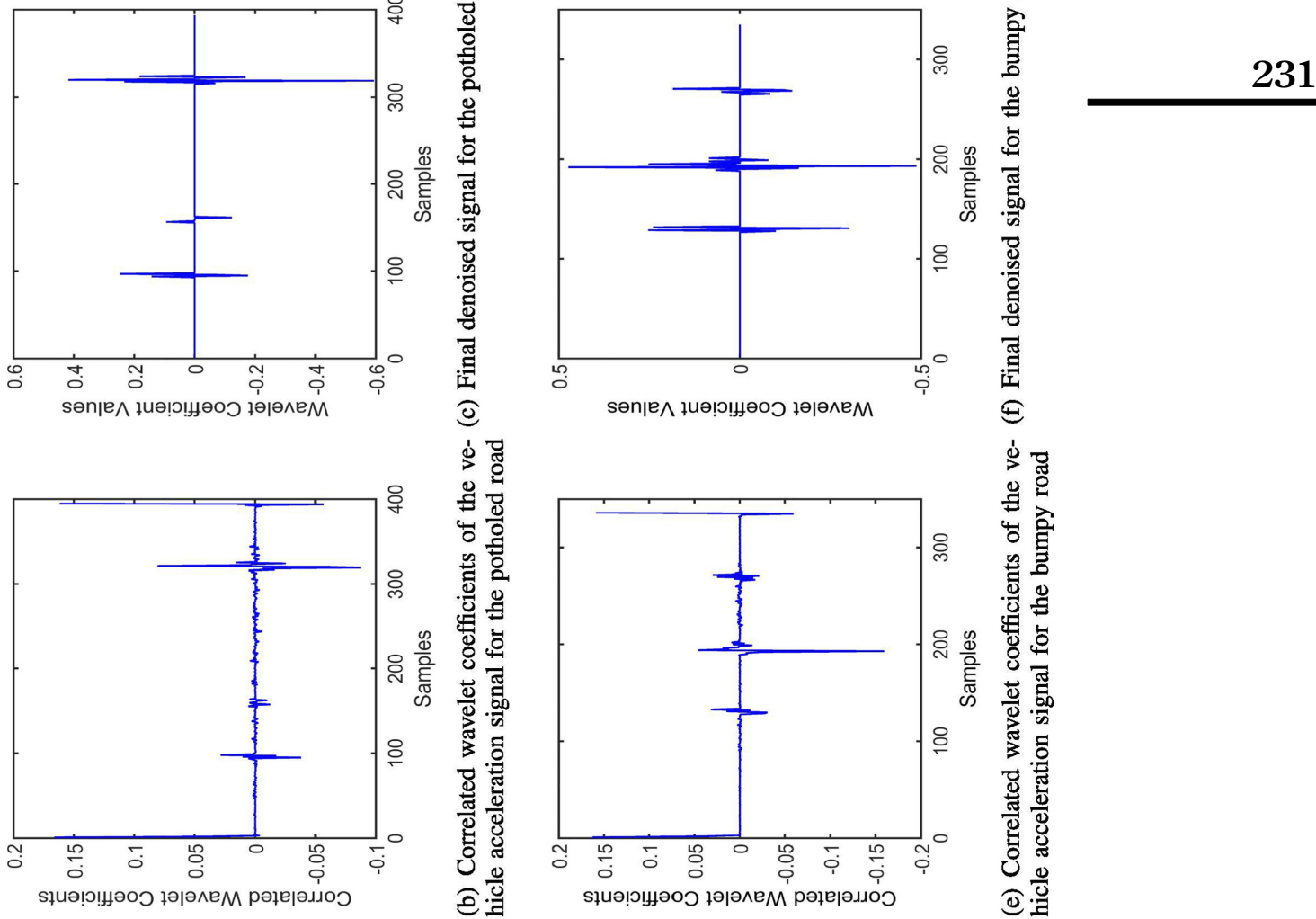


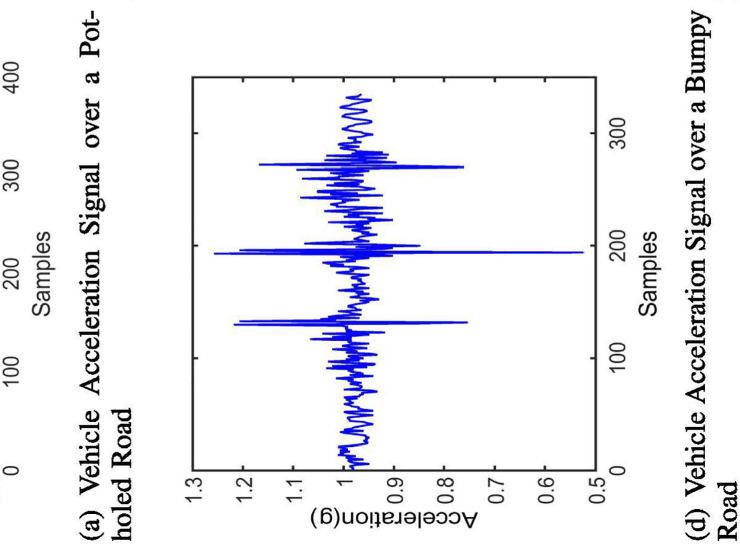

Figure 3. Outputs at different stages of the characterization process. 


\section{$\mathrm{ACI}$ \\ $16,1 / 2$}

\section{2}

Figure 4.

Features representing road anomalies.

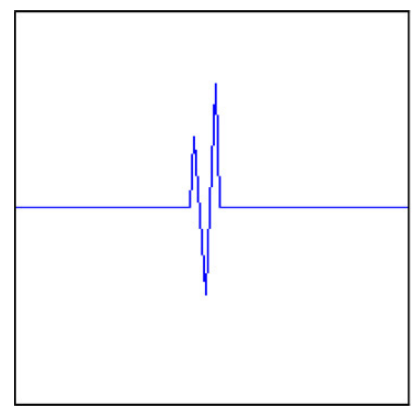

(a) Road Pothole Pattern

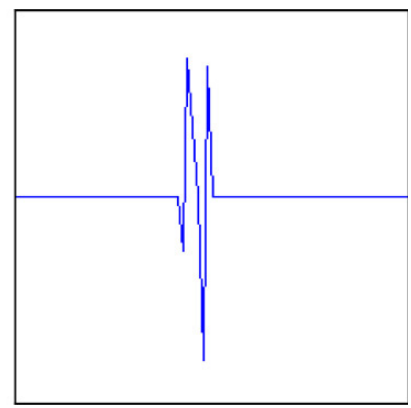

(b) Road Bump Pattern

\subsection{Results of feature extraction}

RACA produced unique features that we used to characterize road anomalies from noisy acceleration signals. Several acceleration signal measurement campaigns were conducted across different road terrains using different vehicles. The performance of the developed SSF, RADA and RACA were tested on new datasets which were not used during the training process. However, for the sake of space, we present two illustrative examples showing acceleration signals acquired over pothole and bumpy road surfaces in Figure 3(a) and (d), respectively. Figure 3(a) represents vehicle acceleration signals acquired over an asphalt road containing only potholes, while Figure 3(d) represents acceleration measurements from an asphalt road having only bumps. First, we show results from the SSF algorithm revealing the correlated wavelet coefficient outputs in Figure 3(b) and (e) for the pothole and bump road surfaces, respectively. We see that these coefficients represent a finer version of the noisy measurements shown in Figure 3(a) and (d), respectively. These were further processed by the SSF algorithm and denoised to produce the final outputs shown in Figure 3(c) and (f), for the pothole and bump road surfaces, respectively. These outputs provide two unique features shown in Figure 4(a) and (b) for characterizing potholes and bumps, respectively. The basis for these features can be explained as follows: a vehicle typically descends into a pothole before ascending out of it, while ascending over a bump before descending down the bump. These different movement patterns are reflected in the acceleration signal which RACA clearly detects by leveraging on the SSF algorithm. RACA uses these features (see Figure 4(a) and (b)) to characterize road anomalies and its performance across different measurements is presented in the next subsections.

Figure 5.

Confusion matrix.

\section{Actual Road Anomalies}

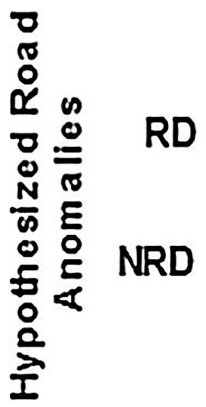

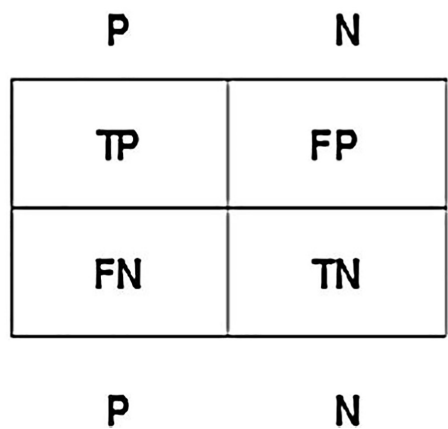


Type of Data: Potholes

Overall Performance Analysis

\begin{tabular}{|c|c|c|c|c|c|}
\hline Threshold & Acc & Prec & FPR & Det. & B \\
\hline 0.0000 & 0.9133 & 0.4878 & 0.0585 & 13 & 1 \\
\hline 0.0010 & 0.9413 & 0.6667 & 0.0279 & 9 & 1 \\
\hline 0.0020 & 0.9439 & 0.6897 & 0.0251 & 8 & 0 \\
\hline 0.0030 & 0.9439 & 0.6897 & 0.0251 & 7 & 1 \\
\hline 0.0040 & 0.9541 & 0.8947 & 0.0056 & 5 & 1 \\
\hline 0.0050 & 0.9413 & 0.9167 & 0.0028 & 4 & 1 \\
\hline 0.0060 & 0.9413 & 0.9167 & 0.0028 & 3 & 0 \\
\hline 0.0070 & 0.9413 & 0.9167 & 0.0028 & 3 & 0 \\
\hline 0.0080 & 0.9413 & 0.9167 & 0.0028 & 3 & 0 \\
\hline 0.0090 & 0.9413 & 0.9167 & 0.0028 & 3 & 0 \\
\hline 0.0100 & 0.9413 & 0.9167 & 0.0028 & 3 & 0 \\
\hline 0.0110 & 0.9413 & 0.9167 & 0.0028 & 3 & 0 \\
\hline 0.0120 & 0.9413 & 0.9167 & 0.0028 & 3 & 0 \\
\hline 0.0130 & 0.9439 & 1.0000 & 0.0000 & 2 & 0 \\
\hline 0.0140 & 0.9439 & 1.0000 & 0.0000 & 2 & 0 \\
\hline 0.0150 & 0.9439 & 1.0000 & 0.0000 & 2 & 0 \\
\hline 0.0160 & 0.9439 & 1.0000 & 0.0000 & 2 & 0 \\
\hline 0.0170 & 0.9337 & 1.0000 & 0.0000 & 2 & 0 \\
\hline 0.0180 & 0.9337 & 1.0000 & 0.0000 & 2 & 0 \\
\hline 0.0190 & 0.9337 & 1.0000 & 0.0000 & 2 & 0 \\
\hline
\end{tabular}

LEGEND: Acc $=$ Accuracy, Prec $=$ Precision.

FPR $=$ False Positive Rate.

Det $=$ Number of Detected Anomalies

$\mathrm{B}=$ Number of Characterized bumps.

$\mathrm{PH}=$ Number of characterized Potholes.

Table 1.

Performance analysis for potholes.

Essentially, for an already labeled dataset, if an anomaly exists (which we refer to as a positive (P)), and our algorithm successfully detects and characterizes this anomaly to be either a pothole or a bump (RD), then a True Positive (TP) is declared. However, if detected to be a non-anomaly $(\mathrm{NRD})$, then a false negative $(\mathrm{FN})$ is declared. Alternatively, if the labeled sample is a non-anomaly $(\mathrm{N})$, and our algorithm detects and characterizes such a sample to be non-anomaly (NRD), then a True Negative (TN) is declared, otherwise, a False Positive (FP) is declared. The equations describing these metrics are as follows:

$$
\begin{aligned}
\text { Accuracy } & =\frac{T P+T N}{P+N} \\
\text { Precision } & =\frac{T P}{T P+F P} \\
F P R & =\frac{F P}{N}
\end{aligned}
$$

\subsection{Performance analysis}

The performances of RADA and RACA were analysed using the following metrics: Accuracy, Precision, and False positive rate (FPR) [29-31]. We describe how these metrics were computed relative to the type of measurements considered in this paper. We considered the confusion matrix shown in Figure 5.

We examined the effect of setting an appropriate detection threshold for the algorithm. The results obtained over different threshold values are provided in Tables 1 and 2. The 
$\mathrm{ACI}$

$16,1 / 2$
Type of Data: Bump

Overall Algorithm's Performance Analysis

\begin{tabular}{lcccccr}
\hline Threshold & Acc & Prec & FPR & Det. & B & PH \\
0.0000 & 0.9286 & 0.4375 & 0.0570 & 11 & 1 & 10 \\
0.0010 & 0.9405 & 0.5000 & 0.0380 & 7 & 0 & 7 \\
0.0020 & 0.9405 & 0.5000 & 0.0380 & 5 & 0 & 5 \\
0.0030 & 0.9583 & 0.6875 & 0.0158 & 4 & 1 & 3 \\
0.0040 & 0.9583 & 0.6875 & 0.0158 & 3 & 2 & 1 \\
0.0050 & 0.9583 & 0.6875 & 0.0158 & 3 & 2 & 1 \\
0.0060 & 0.9583 & 0.6875 & 0.0158 & 3 & 2 & 1 \\
0.0070 & 0.9554 & 0.6667 & 0.0158 & 3 & 2 & 1 \\
0.0080 & 0.9554 & 0.6667 & 0.0158 & 3 & 2 & 1 \\
0.0090 & 0.9554 & 0.6667 & 0.0158 & 3 & 3 & 0 \\
0.0100 & 0.9643 & 0.8333 & 0.0063 & 3 & 3 & 0 \\
0.0110 & 0.9643 & 0.8333 & 0.0063 & 3 & 3 & 0 \\
0.0120 & 0.9643 & 0.8333 & 0.0063 & 3 & 3 & 0 \\
0.0130 & 0.9643 & 0.8333 & 0.0063 & 3 & 3 & 0 \\
0.0140 & 0.9673 & 1.0000 & 0.0000 & 3 & 3 & 0 \\
0.0150 & 0.9673 & 1.0000 & 0.0000 & 3 & 3 & 0 \\
0.0160 & 0.9673 & 1.0000 & 0.0000 & 3 & 3 & 0 \\
0.0170 & 0.9673 & 1.0000 & 0.0000 & 3 & 3 & 0 \\
0.0180 & 0.9673 & 1.0000 & 0.0000 & 3 & 2 & 0 \\
0.0190 & 0.9673 & 1.0000 & 0.0000 & 3 & 2 &
\end{tabular}

LEGEND: Acc $=$ Accuracy, Prec $=$ Precision.

FPR $=$ False Positive Rate.

Table 2.

Average performance analysis for bumps.
Det $=$ Number of Detected Anomalies.

$\mathrm{B}=$ Number of Characterized bumps.

$\mathrm{PH}=$ Number of characterized Potholes.
Table 3.

Effect of using different Daubechy functions, for threshold $=0.01$.

\begin{tabular}{lcccccc}
\hline Acc & Prec & FPR & Def. & B & PH & Wname \\
\hline 0.9413 & 0.9167 & 0.0028 & 3 & 0 & 3 & $\mathrm{db} 2$ \\
0.9286 & 0.8571 & 0.0028 & 2 & 1 & 1 & $\mathrm{db3}$ \\
0.9311 & 1.0000 & 0.0000 & 3 & 2 & 1 & $\mathrm{db} 4$ \\
0.9260 & 0.7500 & 0.0056 & 4 & 1 & 3 & $\mathrm{db5}$ \\
0.9158 & 0.5000 & 0.0084 & 3 & 1 & 2 & $\mathrm{db6}$ \\
0.9056 & 0.0000 & 0.0111 & 3 & 0 & 3 & $\mathrm{db} 7$ \\
0.9031 & 0.2222 & 0.0195 & 3 & 0 & 3 & $\mathrm{db} 8$ \\
0.8827 & 0.0667 & 0.0390 & 3 & 0 & 3 & $\mathrm{db} 9$ \\
0.8801 & 0.0000 & 0.0390 & 3 & 0 & 3 & $\mathrm{db} 10$ \\
0.8954 & 0.1667 & 0.0279 & 3 & 2 & 1 & $\mathrm{db} 11$ \\
0.8878 & 0.0769 & 0.0334 & 3 & 1 & 2 & $\mathrm{db} 12$ \\
0.8801 & 0.0000 & 0.0390 & 3 & 2 & 1 & $\mathrm{db} 13$ \\
0.8903 & 0.0000 & 0.0279 & 3 & 2 & 1 & $\mathrm{db} 14$ \\
0.8801 & 0.1111 & 0.0446 & 3 & 0 & 3 & $\mathrm{db} 15$ \\
0.8750 & 0.0556 & 0.0474 & 3 & 0 & 3 & $\mathrm{db} 16$ \\
0.8571 & 0.0000 & 0.0641 & 3 & 0 & 3 & $\mathrm{db} 17$ \\
0.8827 & 0.0000 & 0.0362 & 3 & 0 & 3 & $\mathrm{db} 18$ \\
0.8673 & 0.0000 & 0.0529 & 3 & 0 & 3 & $\mathrm{db} 19$ \\
\hline
\end{tabular}


algorithm's detection threshold was increased iteratively from 0.000 (minimum threshold) to 0.019 incrementally by 0.001 . An appropriate threshold was identified at the point where no change occured across the different performance metrics. This occured at a threshold value of 0.006 for potholes (see Table 1) leading to an accuracy of $94 \%$, a precision of $92 \%$, and a $0.3 \%$ false positive rate. For bumps, it occured at a threshold value of 0.01 leading to an accuracy of $96 \%$, a precision of $83 \%$, and a very low false positive rate of $0.6 \%$ (see Table 2). Thus, in the absence of an adaptive mechanism, a single threshold value was chosen at 0.01 , which tends to produce the best performance for both pothole and bump features (see Tables 1 and 2). This value was maintained for the rest of the analyses reported in this paper.

\subsection{Choice of algorithm's parameter values}

We examined the performance of our algorithm over a wide range of different parameter values. The results obtained are presented in the following subsections.

4.3.1 Choice of mother wavelet function. We mentioned in Section III-B-2 that different wavelet functions were examined, and the results from these analyses are presented. The different wavelet functions considered are denoted under the variable 'Wname' and the performance results for the family of Daubechy functions is provided in Table 3. A

\begin{tabular}{|c|c|c|c|c|c|c|c|}
\hline Acc & Prec & FPR & Def. & B & $\mathrm{PH}$ & Wname & \\
\hline 0.9388 & 1.0000 & 0.0000 & 2 & 1 & 1 & Coif1 & Table 4 \\
\hline 0.9362 & 0.7857 & 0.0084 & 3 & 0 & 3 & Coif2 & Effect of different \\
\hline 0.9260 & 0.6429 & 0.0139 & 3 & 0 & 3 & Coif3 & Coiflet mother \\
\hline 0.9133 & 0.4667 & 0.0223 & 3 & 0 & 3 & Coif4 & wavelets using \\
\hline 0.9082 & 0.3846 & 0.0223 & 3 & 0 & 3 & Coif5 & threshold $=0.01$ \\
\hline
\end{tabular}

\begin{tabular}{|c|c|c|c|c|c|c|c|}
\hline Acc & Prec & FPR & Def. & $\mathrm{B}$ & $\mathrm{PH}$ & Wname & \\
\hline 0.9413 & 0.9167 & 0.0028 & 3 & 0 & 3 & Sym2 & \\
\hline 0.9286 & 0.8571 & 0.0028 & 2 & 1 & 1 & Sym3 & \\
\hline 0.9388 & 1.0000 & 0.0000 & 2 & 1 & 1 & Sym4 & \\
\hline 0.9464 & 1.0000 & 0.0000 & 3 & 1 & 2 & Sym5 & \\
\hline 0.9388 & 1.0000 & 0.0000 & 2 & 1 & 1 & Sym6 & Table 5 \\
\hline 0.9286 & 0.7778 & 0.0056 & 3 & 1 & 2 & Sym7 & Effect of different \\
\hline 0.9388 & 1.0000 & 0.0000 & 2 & 1 & 1 & Sym8 & Symlet mother \\
\hline 0.9464 & 1.0000 & 0.0000 & 3 & 1 & 2 & Sym9 & wavelets using \\
\hline 0.9388 & 1.0000 & 0.0000 & 2 & 0 & 2 & Sym10 & threshold $=0.01$. \\
\hline
\end{tabular}

\begin{tabular}{lcccccrr}
\hline Acc & Prec & FPR & Def & B & PH & Level & Effect of different \\
\hline 0.9413 & 0.9167 & 0.0028 & 3 & 0 & 3 & 2 & Table 6. \\
0.9413 & 0.9167 & 0.0028 & 4 & 0 & 3 & 4 & decomposition levels \\
0.9056 & 0.7500 & 0.0056 & 5 & 0 & 5 & using db2 and \\
0.9107 & 1.0000 & 0.0000 & 5 & 0 & 5 & threshold $=0.01$. \\
\hline
\end{tabular}


$\mathrm{ACI}$ $16,1 / 2$

\section{6}

\begin{tabular}{lccccccr}
\cline { 2 - 7 } & Acc & Prec & FPR & Def. & B & PH & Window \\
\cline { 2 - 7 } & 0.9337 & 0.8889 & 0.0028 & 4 & 2 & 2 & 5 \\
Table 7. & 0.9388 & 0.9091 & 0.0028 & 3 & 0 & 3 & 10 \\
Effect of different & 0.9388 & 0.9091 & 0.0028 & 3 & 0 & 3 & 20 \\
window Sizes for & 0.9413 & 0.9167 & 0.0028 & 3 & 0 & 3 & 30 \\
Wname = db2, and & 0.9388 & 0.9091 & 0.0028 & 3 & 0 & 3 & 40 \\
threshold =0.01. & 0.9413 & 0.9167 & 0.0028 & 3 & 0 & 3 & 50 \\
\hline
\end{tabular}

Figure 6.

Profiled road.

combination of the measurements for both potholes and bumps was used. Furthermore, the optimal threshold value of 0.01 (see Section B above) was used. We observed (see Table 3 ) that accuracy and precision decreased for higher members of the Daubechy family (db2 to db19), while false alarm increased. Thus, lower members of the family of Daubechy functions performed better than higher members. The $\mathrm{db} 2$ mother wavelet function was selected to be the best choice for the SSF algorithm leading to an average accuracy of $94 \%$, precision of $92 \%$, and a false alarm rate of $0.3 \%$ respectively.

Other wavelet functions were examined such as the Coiflet (Coif) and Symlet (Sym) functions and results obtained are presented in Table 4 and 5, respectively. We observed

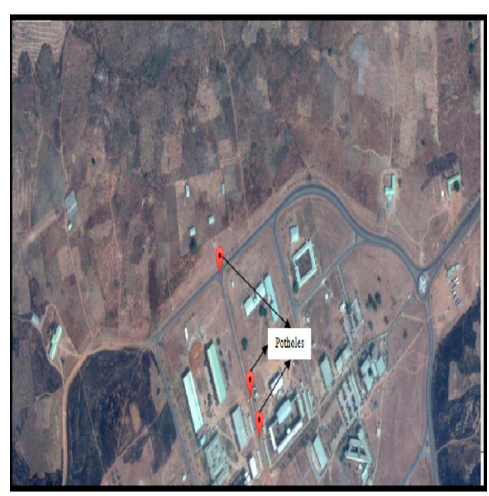

(a) Profiled Road showing Pothole Labels

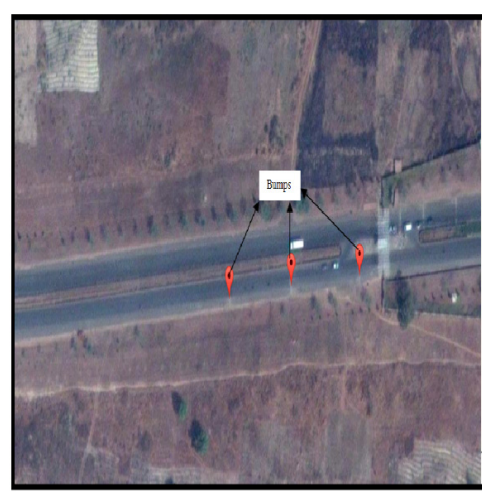

(b) Profiled Road showing Pothole Labels
Table 8.

Corresponding GPS coordinates of road anomalies location.

\begin{tabular}{lcr}
\hline Road Anomalies & Longitude & Latitude \\
\hline Pothole 1 & 6.449853 & 9.534304 \\
Pothole 2 & 6.449581 & 9.534817 \\
Pothole 3 & 6.448710 & 9.536412 \\
Bump 1 & 6.464269 & 9.536809 \\
Bump 2 & 6.464707 & 9.536852 \\
Bump 3 & 6.465189 & 9.536881 \\
\hline
\end{tabular}


that the Coiflet function produced high precision and low false alarm rates at the expense of poor characterization performances (see the B and $\mathrm{PH}$ values in Table 4). For higher members of the Coiflet wavelet family, our SSF algorithm was able to detect and characterize appropriately with low accuracy and precision rates (see Table 4) as compared to the db2 function in Table 3. Moreover, observe in Table 5 that the Sym2 wavelet function produced the same accuracy, precision, and false alarm rate with the $\mathrm{db} 2$ function in Table 3. This implies that either of these two mother wavelets can be used to achieve good performance. The db2 function was chosen for use in our SSF algorithm. Other wavelet functions such as the Mexican Hat (Morlet), Meyer and Shannon functions were not considered because of their dissimilar pattern to the acceleration signals considered in this work.

4.3.2 Performance under varying decomposition levels. We studied the effect of the number of decomposition levels on the performance of RACA. Different WT decomposition levels in the SSF algorithm were examined and results obtained are provided in Table 6 . We observed (see Table 6) that at higher decomposition levels (specifically above 3), RACA performed poorly. One reason we may suggest is that at higher levels, the higher frequency components of the signal are filtered out leading to the loss of important signal features. Consequently, two decomposition levels were considered in the SSF algorithm to improve RACA's performance.

4.3.3 Performance under varying window size. The window size in RACA aggregates a number of samples within which an anomaly can be detected. Thus, RACA's performance was examined with regards to different window sizes. The $\mathrm{db} 2$ wavelet function was used (being the best choice) and a window size ranging from 10 and 50 samples produced an appreciable performance level (see Table 7). However, the algorithm performed poorly with window sizes below 5 samples. Therefore, a window size of 30 samples was considered suitable for RACA to guarantee high accuracy, precision and lower FPR values. In addition, higher values may only increase the required memory size, and not necessarily improve the algorithm's performance.

4.3.4 The road profiler output. We provide a visual feel of the output of the profiler showing both potholes and bumps in Figure 6(a) and (b), respectively. This was done for a few sampled anomalies having their respective GPS coordinates stated in Table 8 . The red ${ }^{1}$ marks in Figure 6(a) serve to indicate the different locations of the detected and characterized anomalies. These maps in Figure 6(a) and (b) aim to demonstrate the concept and functionality of the road profiler system. Nevertheless, further development in this regard is required. The profiler's output can be used by drivers to identify defective portions along the road surface towards improving the driving process.

\section{Conclusion}

This paper has presented algorithms for the detection and characterization of road anomalies for possible deployment in both manned and unmanned vehicles. Our algorithm works by filtering noisy measurements obtained via an Accelerometer mounted in a vehicle. The positive correlation property across different scales in the WT domain is employed to detect anomalous points in the acceleration signal. Two unique features were developed to distinguish potholes from bumps. Results obtained suggest that these features can be successfully used to characterize road anomalies with a high level of accuracy, precision and a low false positive rate. Future works will focus on developing adaptive mechanisms to vary the threshold value of the algorithm based on different measurement sets rather than on a static threshold value considered at this point of our research. In addition, mechanisms for communicating the characterized road anomaly information among vehicles or between a vehicle and a road side infrastructure in typical VANET systems will be examined. At the moment, it is noted that WT processors may be unavailable as off-the-shelve chipsets, thus 
$\mathrm{ACI}$

$16,1 / 2$

making the practical development of our approach an interesting issue for future research consideration. Our current research contributes towards improving road anomaly characterization for future deployment in autonomous vehicles.

\section{Notes}

1 For interpretation of color in Figure 6, the reader is referred to the web version of this article.

\section{8}

\section{References}

[1] G. Alessandroni, L. Klopfenstein, S. Delpriori, M. Dromedari, G. Luchetti, B. Paolini, A. Seraghiti, E. Lattanzi, V. Freschi, A. Carini, et al., Smartroadsense: collaborative road surface condition monitoring, in: Proceedings of the UBICOMM, 2014, pp. 210-215.

[2] H. Bello-Salau, A. Aibinu, E. Onwuka, J. Dukiya, M. Bima, A. Onumanyi, T. Folorunso, A new measure for analysing accelerometer data towards developing efficient road defect profiling systems, , J. Scient. Res. Rep. 7 (2) (2015) 108-116.

[3] O. Agbonkhese, G. Yisa, E. Agbonkhese, D. Akanbi, E. Aka, E. Mondigha, Road traffic accidents in Nigeria: causes and preventive measures, Civil Environ. Res. 3 (13) (2013) 90-99.

[4] A.F. Sumaila, Road crashes trends and safety management in Nigeria, J. Geogr. Regional Plan. 6 (3) (2013) 53.

[5] O.O. Lanre, Optimizing post-construction lifeline of the Nigerian road network system: failures and causes, preventions and remedies, J. Eng. Appl. Sci. 1 (4) (2006) 456-461.

[6] J.-P. Rodrigue, C. Comtois, and B. Slack, The geography of transport systems, Taylor \& Francis, 2016.

[7] H. Bello-Salau, A.M. Aibinu, E.N. Onwuka, J.J. Dukiya, and A.J. Onumanyi, Image processing techniques for automated road defect detection: a survey, in: 2014 11th International Conference on Electronics, Computer and Computation (ICECCO), IEEE, 2014, pp. 1-4.

[8] H. Bello-Salau, A.M. Aibinu, E.N. Onwuka, J.J. Dukiya, A.J. Onumanyi, A.O. Ighabon, Development of a laboratory model for automated road defect detection, J. Telecommun., Electron. Comput. Eng. (JTEC) 8 (9) (2016) 97-101.

[9] G. Alessandroni, L. Klopfenstein, S. Delpriori, M. Dromedari, G. Luchetti, B. Paolini, A. Seraghiti, E. Lattanzi, V. Freschi, A. Carini, et al., Smartroadsense: collaborative road surface condition monitoring, Proc. UBICOMM (2014) 210-215.

[10] V. Astarita, M.V. Caruso, G. Danieli, D.C. Festa, V.P. Giofrè, T. Iuele, R. Vaiana, A mobile application for road surface quality control: uniqualroad, , Proc. - Soc. Behav. Sci. 54 (2012) $1135-1144$.

[11] V. Douangphachanh, H. Oneyama, A study on the use of smartphones for road roughness condition estimation, J. East. Asia Soc. Transport. Stud. 10 (2013) 1551-1564.

[12] R.S. Figliola and D. Beasley, Theory and design for mechanical measurements, John Wiley \& Sons, 2015.

[13] Y. c. Tai, C.W. Chan, and J.Y.J. Hsu, Automatic road anomaly detection using smart mobile device, in: Conference on Technologies and Applications of Artificial Intelligence, Hsinchu, Taiwan, 2010.

[14] A. Mohamed, M.M.M. Fouad, E. Elhariri, N. El-Bendary, H.M. Zawbaa, M. Tahoun, A.E. Hassanien, Roadmonitor: an intelligent road surface condition monitoring system, in: Intelligent Systems' 2014, Springer, 2015, pp. 377-387.

[15] F. Seraj, B.J. van der Zwaag, A. Dilo, T. Luarasi, P. Havinga, Roads: a road pavement monitoring system for anomaly detection using smart phones, in: International Workshop on Modeling Social Media, Springer, 2014, pp. 128-146.

[16] L.C. González-Gurrola, F. Martínez-Reyes, M.R. Carlos-Loya, The citizen road watcher-identifying roadway surface disruptions based on accelerometer patterns., in: UCAmI, Springer, 2013, pp. 374-377. 
[17] M. Perttunen, O. Mazhelis, F. Cong, M. Kauppila, T. Leppänen, J. Kantola, J. Collin, S. Pirttikangas, J. Haverinen, T. Ristaniemi, et al., Distributed road surface condition monitoring using mobile phones, in International Conference on Ubiquitous Intelligence and Computing, Springer, 2011, pp. 64-78.

[18] M. Strutu, G. Stamatescu, and D. Popescu, A mobile sensor network based road surface monitoring system, in System Theory, Control and Computing (ICSTCC), 2013 17th International Conference, IEEE, 2013, pp. 630-634.

[19] J. Eriksson, L. Girod, B. Hull, R. Newton, S. Madden, H. Balakrishnan, The pothole patrol: using a mobile sensor network for road surface monitoring, in: Proceedings of the 6th International Conference on Mobile Systems, Applications, and Services, ACM, 2008, pp. 29-39.

[20] R. Hesami, K.J. McManus, Signal processing approach to road roughness analysis and measurement, in: TENCON 2009-2009 IEEE Region 10 Conference, IEEE, 2009, pp. 1-6.

[21] C.-W. Yi, Y.-T. Chuang, C.-S. Nian, Toward crowdsourcing-based road pavement monitoring by mobile sensing technologies, IEEE Trans. Intell. Transport. Syst. 16 (4) (2015) 1905-1917.

[22] J. Dawkins, R. Bishop, B. Powell, and D. Bevly, Investigation of pavement maintenance applications of intellidrive sm (final report): implementation and deployment factors for vehicle probe-based pavement maintenance (pbpm), 2011.

[23] G. Strazdins, A. Mednis, G. Kanonirs, R. Zviedris, L. Selavo, Towards vehicular sensor networks with android smartphones for road surface monitoring, in 2nd international workshop on networks of cooperating objects, Chicago, USA, 2011.

[24] A. Vittorio, V. Rosolino, I. Teresa, C.M. Vittoria, P.G. Vincenzo, et al., Automated sensing system for monitoring of road surface quality by mobile devices, , Proc. - Soc. Behav. Sci. 111 (2014) 242-251.

[25] A. Mednis, G. Strazdins, R. Zviedris, G. Kanonirs, L. Selavo, Real time pothole detection using android smartphones with accelerometers, in: Distributed Computing in Sensor Systems and Workshops (DCOSS), 2011 International Conference on, IEEE, 2011, pp. 1-6.

[26] H. Ohize, A. Onumanyi, M. Dlodlo, and H. Bello-Salau, An adaptive wavelet-based scale space filtering algorithm for spectrum sensing in cognitive radio, in: Wireless Communications and Networking Conference (WCNC), 2017 IEEE, IEEE, 2017, pp. 1-6.

[27] Y. Xu, J.B. Weaver, D.M. Healy, J. Lu, Wavelet transform domain filters: a spatially selective noise filtration technique, IEEE Trans. Image Process. 3 (6) (1994) 747-758.

[28] O.C. Ugweje, Selective noise filtration of image signals using wavelet transform, Measurement 36 (3) (2004) 279-287.

[29] T. Fawcett, An introduction to ROC analysis, Pattern Recogn. Lett. 27 (8) (2006) 861-874.

[30] A.J. Onumanyi, E.N. Onwuka, A.M. Aibinu, O.C. Ugweje, M.J.E. Salami, A modified otsus algorithm for improving the performance of the energy detector in cognitive radio, AEU-Int. J. Electron. Commun., 2017.

[31] A.J. Onumanyi, E.N. Onwuka, A.M. Aibinu, O.C. Ugweje, M.J.E. Salami, A real valued neural network based autoregressive energy detector for cognitive radio application, Int. Scholar. Res. Notices 2014 (2014) 1-11.

\section{Corresponding author}

H. Bello-Salau can be contacted at: habeeb.salau@futminna.edu.ng

For instructions on how to order reprints of this article, please visit our website:

www.emeraldgrouppublishing.com/licensing/reprints.htm

Or contact us for further details: permissions@emeraldinsight.com 